\title{
Carbapenem-resistant Acinetobacter baumannii: Colonization, Infection and Current Treatment Options
}

\author{
Carmi Bartal · Kenneth V. I. Rolston · Lior Nesher (1)
}

Received: December 22, 2021 / Accepted: January 25, 2022 / Published online: February 17, 2022

(C) The Author(s) 2022

\begin{abstract}
Carbapenem-resistant Acinetobacter baumannii (CRAB) causes colonization and infection predominantly in hospitalized patients. Distinction between the two is a challenge. When CRAB is isolated from a non-sterile site (soft tissue, respiratory samples, etc.), it probably represents colonization unless clear signs of infection (fever, elevated white blood count, elevated inflammatory markers and abnormal imaging) are present. Treatment is warranted only for true infections. In normally sterile sites (blood, cerebrospinal fluid) the presence of indwelling medical devices (catheters, stents) should be considered when evaluating positive cultures. In the absence of such devices, the isolate represents an infection and should be
\end{abstract}

C. Bartal · L. Nesher $(\bowtie)$

Faculty of Health Sciences, Internal Medicine, Soroka Medical Center, Ben-Gurion University of the Negev, Beer Sheba, Israel

e-mail: nesherke@bgu.ac.il

\section{K. V. I. Rolston}

The Department of Infectious Diseases, Infection Control, and Employee Health, Unit 1460, The University of Texas MD Anderson Cancer Center, Houston, TX, USA

\section{Nesher}

Faculty of Health-Sciences, Infectious Disease Institute, Soroka Medical Center, Ben-Gurion University of the Negev, 1 Rager Street, Beer-Sheba, Israel treated. If an indwelling device is present and there are no signs of active infection, the device should be replaced if possible, and no treatment is required. If there are signs of an active infection the device should be removed or replaced, and treatment should be administered. Current treatments options and clinical data are limited. No agent or combination regimen has been shown to be superior to any other in randomized clinical trials. Ampicillin-sulbactam appears to have the best evidence for initial use. This is probably due to its ability to saturate penicillin-binding proteins 1 and 3 when given in high dose. Tigecycline when used should be given in high dose as well. Polymyxins are a treatment option but are difficult to dose correctly and have significant side effects. Newer treatment options such as eravacycline and cefiderocol have potential; however, currently there are not enough data to support their use as single agents. Combination therapy appears to be the best treatment option and should always include high-dose ampicillin-sulbactam combined with another active agent such as high-dose tigecycline, polymyxins, etc. These infections require a high complexity of skill, and an infectious disease specialist should be involved in the management of these patients.

Keywords: Acinetobacter infections; Drug resistance; Multiple; Carbapenem-resistant enterobacteriaceae 


\section{Key Summary Points \\ Isolating carbapenem-resistant Acinetobacter Baumannii (CRAB) in a patient may represent colonization or infection; distinguishing between the two is challenging \\ CRAB isolated from non-sterile sites or from sterile sites that have an indwelling device without signs of infection represents colonization and should not be treated (see Fig. 1) \\ Current treatment options and clinical data are limited. No agent or combination regimen has been shown to be superior to any other in randomized clinical trials \\ Ampicillin-sulbactam appears to have the best evidence for initial use. This is probably due to its ability to saturate penicillin-binding proteins 1 and 3 when given in high dose \\ Combination therapy appears to be the best treatment option and should always include high-dose ampicillin-sulbactam combined with another active agent such as high-dose tigecycline, polymyxins or one of the other newer agents (cefiderocol, eravacycline) (see Table 1)}

\section{INTRODUCTION}

Carbapenem-resistant Acinetobacter baumannii (CRAB) cause significant life-threating hospital-acquired infections. In the World Health Organization (WHO) priority pathogens list for research and development of new antibiotics, CRAB has been designated a priority 1-critical pathogen [1]. It poses a significant therapeutic challenge due to a lack of established treatment options because very few currently available antimicrobial agents are active against them. Globally, ampicillin-sulbactam is considered the only agent for monotherapy against these organisms. Most other therapeutic choices include various other agents combined with ampicillin-sulbactam. These organisms frequently colonize hospitalized patients, particularly those on ventilators or receiving care in intensive care units, and it is often difficult to distinguish colonization from infection. In this narrative review we attempt to provide clarification on the issues including colonization, infection and current treatment options. As this review is based on previously conducted studies and peer-reviewed articles, no permission was required from the institutional ethics review board.

\section{EPIDEMIOLOGY}

Acinetobacter is rarely a cause of communityacquired infection. It is frequently found in water and soil, and in humans it can colonize various sites including skin, respiratory and gastrointestinal tracts [2]. Acinetobacter accounts for approximately $2 \%$ of nosocomial infections in the USA but these rates are doubled in Asia and the Middle East with up to $20 \%$ of infections in intensive care units (ICU) worldwide [3, 4]. In most institutions the majority of Acinetobacter isolated is not CRAB; however, it frequently causes outbreaks and becomes a major concern for infection control. The main characteristics implicated in Acinetobacter pathogenicity are its capability to survive environmental desiccation for weeks promoting transmission through fomite contamination in hospitals [5]. Several virulence factors have been identified in CRAB but the details are outside the scope of our review.

Most information about health care-associated Acinetobacter infections is based on data from outbreak investigations, and complex patients in the ICU setting are considered to be at the highest risk [6]. The mortality rate of Acinetobacter infection is high ranging from 45 to $70 \%$ [7-9]. A meta-analysis found that 
carbapenem resistance is the main predictor of mortality [8].

\section{CLINICAL SYNDROMES}

Pneumonia is the primary manifestation of CRAB; approximately 55\% of CRAB infections involve the respiratory system [10]. It occurs predominantly as ventilator-associated pneumonia (VAP) and tends to have late onset during ICU hospitalization. Most occurs in previously colonized patients. Patients who develop VAP with CRAB require prolonged ventilation and have extended ICU stay compared to patients with VAP caused by other gram-negative bacilli [9]. Acinetobacter accounts for only $2 \%$ of nosocomial blood stream infections. Most are catheter-related infections or complication of respiratory tract infections [11]. Bacteremia and septic shock early in the infection are factors associated with bad prognosis [12]. Skin, soft tissue and bone infections also occur; they usually appear after colonization and in association with surgical interventions or in the presence of infected prosthetic joints [13]. Many soft tissue infections have been reported as war injuries, probably due to field hospital contamination [14].

Other rare sites of infection include endocarditis in either native or prosthetic valves; these often fatal events are associated with a high rate of early valve destruction [15]. Meningitis due to Acinetobacter usually involves neurosurgical procedures such as ventriculostomy or intrathecal administration of chemotherapy and post-surgery CSF leak [16]. Acinetobacter is a common reason for eye infection causing corneal ulcers $(7 \%$ of eye

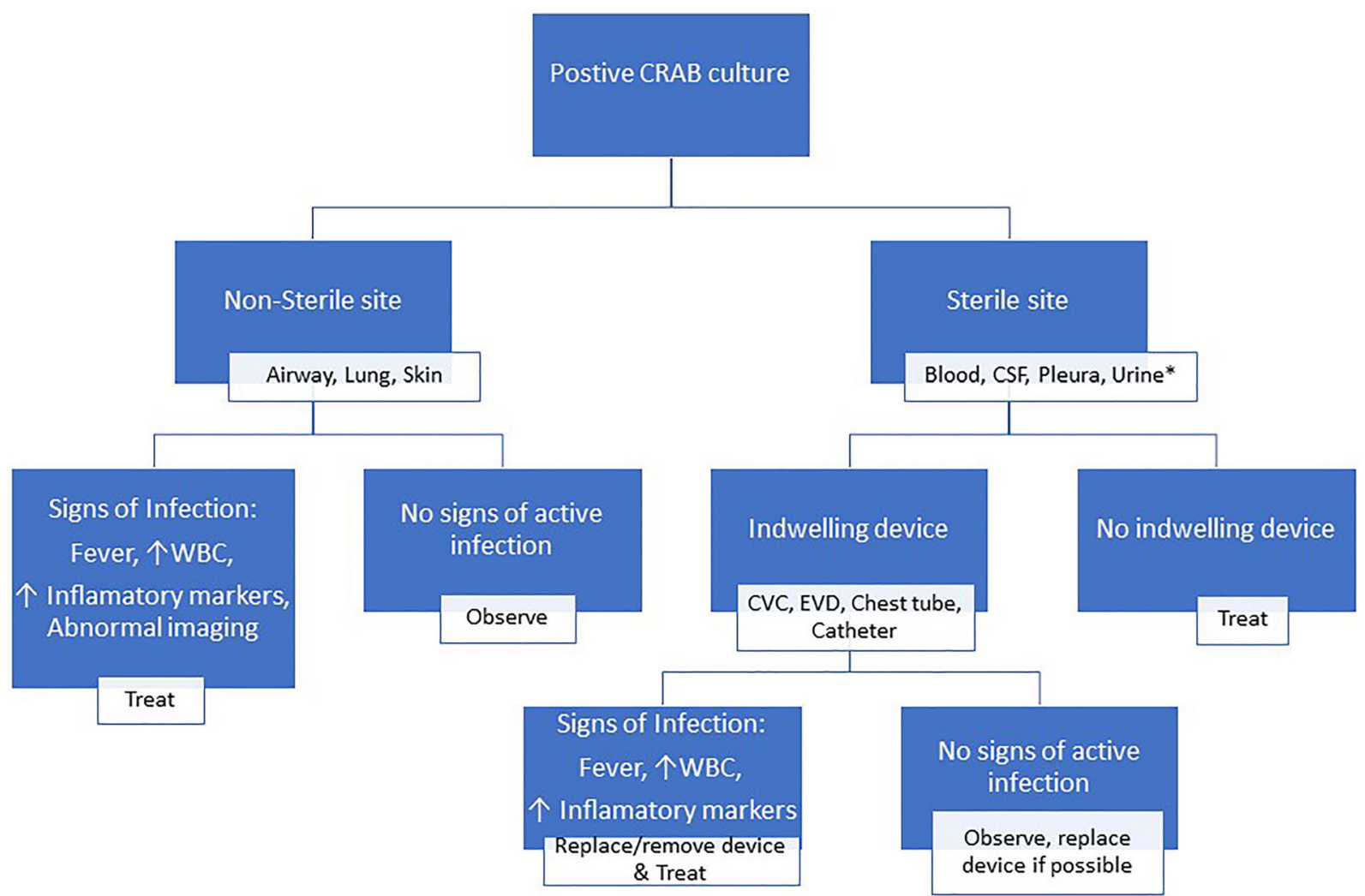

Fig. 1 Management algorithm of patients with a positive CRAB culture. ${ }^{*}$ Most cultures in this setting represent colonization and not infection 
Table 1 Antibiotics used for the treatment of CRAB infections

\begin{tabular}{|c|c|c|c|}
\hline Agent & $\begin{array}{l}\text { Adult dosage (assuming normal renal and liver } \\
\text { function) }\end{array}$ & Remarks & $\begin{array}{l}\text { Major toxicities } \\
\text { to consider }\end{array}$ \\
\hline $\begin{array}{l}\text { aAmpicillin- } \\
\text { sulbactam }\end{array}$ & $\begin{array}{l}3 \mathrm{~g} \text { every } 4 \mathrm{~h} \text { if intolerance or toxicities preclude the use } \\
\text { of higher dosages or for mild infections }\end{array}$ & & $\begin{array}{l}\text { Hepatotoxicity } \\
\quad(1 \%)\end{array}$ \\
\hline $\begin{array}{l}\text { ampicillin- } \\
\text { Sulbactam }\end{array}$ & $\begin{array}{l}9 \mathrm{~g} \text { every } 8 \mathrm{~h} \text {, each dose given over } 4 \mathrm{~h} \\
27-\mathrm{g} \text { continuous infusion over } 24 \mathrm{~h}\end{array}$ & $\begin{array}{l}\text { High dose, suitable for } \\
\text { ampicillin-sulbactam- } \\
\text { resistant CRAB }\end{array}$ & \\
\hline Cefiderocol & $2 \mathrm{~g}$ every $8 \mathrm{~h}$ infused over $3 \mathrm{~h}$ & & $\begin{array}{l}\text { Elevated liver } \\
\text { tests }(2-16 \%) \\
\text { Hypokalemia } \\
(11 \%)\end{array}$ \\
\hline Colistin & As per international consensus guidelines ${ }^{b}$ & & $\begin{array}{l}\text { Nephrotoxicity } \\
\qquad(1-18 \%) \\
\text { Neurotoxicity } \\
(1-7 \%)\end{array}$ \\
\hline Eravacycline & $1 \mathrm{mg} / \mathrm{kg} /$ dose every $12 \mathrm{~h}$ & & GI $(2-7 \%)$ \\
\hline $\begin{array}{l}\text { 'c Imipenem- } \\
\text { cilastatin }\end{array}$ & $500 \mathrm{mg}$ every $6 \mathrm{~h}$ infused over $3 \mathrm{~h}$ & & Seizures $(1 \%)$ \\
\hline${ }^{\mathrm{c}}$ Meropenem & $2 \mathrm{~g}$ every $8 \mathrm{~h}$ infused over $3 \mathrm{~h}$ & & Seizures $(<1 \%)$ \\
\hline Minocycline & $200 \mathrm{mg}$ every $12 \mathrm{~h}$ & & CNS $(1-3 \%)$ \\
\hline Tigecycline & $200 \mathrm{mg}$ once, then $100 \mathrm{mg}$ every $12 \mathrm{~h}$ & High dose & $\begin{array}{l}\text { Hepatotoxicity } \\
\qquad(2-5 \%) \\
\text { Pancreatitis } \\
\quad(<1 \%)\end{array}$ \\
\hline
\end{tabular}

CRAB carbapenem-resistant Acinetobacter baumannii

${ }^{a}$ Currently only ampicillin-sulbactam is considered appropriate for monotherapy; all other drugs should be used based on susceptibly as a combination with ampicillin sulbactam except in penicillin-allergic patients

${ }^{\mathrm{b}}$ Tsuji BT, Pogue JM, Zavascki AP, et al. International Consensus Guidelines for the Optimal Use of the Polymyxins: Endorsed by the American College of Clinical Pharmacy (ACCP), European Society of Clinical Microbiology and Infectious Diseases (ESCMID), Infectious Diseases Society of America (IDSA), International Society for Anti-infective Pharmacology (ISAP), Society of Critical Care Medicine (SCCM), and Society of Infectious Diseases Pharmacists (SIDP). Pharmacotherapy 2019; 39(1): 10-39

${ }^{\mathrm{c}}$ Carbapenems may be considered as a third drug in combination regiments

infections) usually after cataract surgery or other ophthalmic surgical procedures [17].

Urinary tract infection with Acinetobacter is rare. Acinetobacter in the urine is documented predominantly after prolonged urinary catheterization and mostly represents colonization. Fewer than $2 \%$ of patients with Acinetobacter colonization in the urine will developed an invasive infection [11]. 


\section{Colonization Versus Infection}

Differentiating between colonization and infection is challenging. CRAB is commonly recovered from respiratory samples, soft tissue cultures and the urine. Most patients have a long history of exposure to the health care system, have multiple comorbidity factors and may have prolonged ICU stay/ventilation.

We suggest that when CRAB is isolated on culture the first question one should ask is whether it was isolated from a sterile or a non-sterile site (Fig. 1). From a non-sterile site such as the airways, lung or skin, the isolation of CRAB generally represents colonization unless there are clear signs of infection (fever, elevated white blood count, elevated inflammatory markers and abnormal imaging). Treatment is warranted only if there is a high clinical suspicion of infection along with the presence of some signs of infection. Nevertheless, it may be prudent to provide preemptive therapy against CRAB in immunosuppressed or other high-risk patients such as transplant patients, patients with malignancy or those receiving corticosteroids when it is difficult to distinguish between infection and colonization because of suppressed inflammatory response. The presence of indwelling medical devices (catheters, stents, etc.) should be considered when evaluating positive cultures from normally sterile sites such as the blood, cerebral spinal fluid, pleura, etc. If no such devices exist, then the isolate represents an infection and should be treated. If an indwelling device is present and there are no signs of active infection, the device should be replaced if possible and no treatment is needed. If there are signs of an active infection, the device should be removed or replaced and the patient treated. Placement of urinary catheters is commonplace in patients receiving care in intensive care units. Urinary cultures obtained from such patients are frequently positive. However, most positive cultures in the urine of such patients represent colonization and do not require treatment unless there are clear signs of infection of urinary source.

\section{Treatment Options}

Inherent and acquired resistance mechanisms severely limit the antimicrobial options for CRAB. There are a few small, randomized trials that have evaluated the efficacy and safety of antimicrobial agents or combination regimens for the treatment of CRAB. Most evidence available to support different treatment options is observational or non-randomized with limited sample size and immense diversity in the severity of infection and comorbidities. Recently the Infectious Disease Society of America (IDSA) released guidelines for treatment of CRAB[18]; we have summarized their recommendations as well as our opinions of current treatment options. A list of treatment options is shown in Table 1.

\section{Ampicillin-Sulbactam}

Sulbactam is an irreversible competitive beta lactamase inhibitor that has the ability to saturate Penicillin Binding Proteins (PBP) 1 and 3 when given in high doses [19]. This unique method of activity has not been exhibited by other beta lactamase inhibitors and thus has an added benefit for treatment. Ampicillin-sulbactam is delivered as a combination of $2 \mathrm{~g}$ ampicillin and $1 \mathrm{~g}$ sulbactam. High-dose ampicillin-sulbactam, which achieves saturation of PBPs, is considered a dose of $27 \mathrm{~g}$ per day or the equivalent of $9 \mathrm{~g}$ sulbactam either in a continuous infusion or as an extended infusion [18]. In some countries where it is available, cefoperazone/sulbactam may represent an alternative to ampicillin/sulbactam. Non-beta-lactam betalactamase inhibitors such as avibactam, relebactam and vaborbactam appear to have no therapeutic role against CRAB.

Liu et al. have recently published a metaanalysis of 18 studies that included 1835 patients that demonstrated that high-dose ampicillin-sulbactam of at least $18 \mathrm{~g}$ per day in combination with a second agent was the most effective regimen to reduce mortality in critically ill CRAB patients [20]. In 2017, Jung et al. published a meta-analysis of 23 studies 
including 2118 patients that identified ampicillin-sulbactam as having the greatest impact on reducing mortality when compared to polymyxin or tetracycline based regimens [21]. These studies combined with others have convinced the IDSA panel to recommend high-dose ampicillin-sulbactam as the preferred back bone therapy for treatment of lifethreatening CRAB either as a single agent or as part of combination therapy. Furthermore, there is evidence that even though CRAB may demonstrate laboratory non-susceptibility to ampicillin-sulbactam, providing high-dose ampicillin-sulbactam may still be an effective therapy in vivo through the mechanism of PBP saturation [22, 23].

\section{Polymyxins}

Polymyxin's (both B and E) are used as part of the treatment of CRAB. Both polymyxins have reliable in vitro activity against CRAB isolates with most of the published literature focusing on Polymyxin E (Colistin). Colistin has been used with some success for the treatment of CRAB pneumonia, bacteremia and meningitis $[8,24]$. A meta-analysis of six studies with a total of 359 patients comparing the use of colistin to carbapenem or high-dose ampicillin/sulbactam showed a similar safety profile and no difference in clinical outcomes for patients with VAP. However, the study included other multidrug-resistant pathogens including Pseudomonas spp. [25].

Colistin is administered as colisimethate (CMS), a prodrug that needs to be hydrolyzed to its active form. CMS is largely excreted in the urine $(70 \%)$ and is partly transformed to the active form of colistin (30\%), whereas renal excretion of colistin is negligible (1-2\%). As renal function decreases, a progressively larger fraction of CMS will be converted to colistin. Therefore, appropriate dosing of colistin is challenging. The ratio of the area under the curve (AUC) to the minimal inhibitory concentration (MIC) AUC/MIC ratio is the best pharmacokinetic-pharmacodynamic index to describe its efficacy profile [26]. A dosing regimen has been suggested that should allow for colistin plasma concentration of about $2 \mathrm{mg} / \mathrm{l}$ to assure the efficacy against colistin-susceptible CRAB [27].

Nephrotoxicity is the most serious adverse event associated with the use of colistin. In a meta-analysis of 237 reports including a total of 35,569 patients, the nephrotoxicity rate was $39 \%$ [28]. The likelihood of nephrotoxicity was significantly greater with polymyxin therapies compared to non-polymyxin-based regimens (OR 2.23). Factors impacting nephrotoxicity included dose, patient age, number of concomitant nephrotoxins and use of diuretics, glycopeptides or vasopressors [28].

Other limitations to the use of polymyxins include suboptimal activity of colistin in pulmonary epithelial cells resulting in reduced activity in the lungs [29] as well as reports of clinical failure and resistance emergence during polymyxin monotherapy [30, 31].

In conclusion, polymyxins should preferably be used in combination with at least one other agent (ampicillin-sulbactam) for the treatment of CRAB infections.

\section{Minocycline}

Minocycline is available for both intravenous and oral use, exhibits bactericidal activity against $A$. baumannii including CRAB and may act synergistically when combined with other agents such as colistin, rifampin and carbapenems $[32,33]$. IV minocycline holds a US Food and Drug Administration indication for the treatment of infections caused by Acinetobacter. In retrospective studies, the use of IV minocycline provided high rates of clinical success or improvement and was generally well tolerated among patients with CRAB; however, minocycline was provided in combination with other antibiotics and not as a single agent and was recommended to be given as a high dose of $200 \mathrm{mg}$ every $12 \mathrm{~h}[33,34]$.

\section{Tigecycline}

Tigecycline is a derivative of minocycline and is the first member of the glycylcycline class [35]. In general, tigecycline should not be used as a 
single agent when other effective antibiotic choices are available. Tigecycline has appealing activity against MDR pathogens except for Pseudomonas aeruginosa and Proteus spp. Furthermore, it has activity against CRAB, although resistance has been reported and clinical experience is limited with conflicting reports for and against its use [36, 37].

As there is no Clinical and Laboratory Standards Institute (CLSI) breakpoint for Acinetobacter baumannii, there is no meaningful breakpoint to determine susceptibility. A higher dose of tigecycline (200 mg intravenous loading dose followed by $100 \mathrm{mg}$ every $12 \mathrm{~h}$ ) showed no mortality differences between tigecycline and comparator agents, and intrapulmonary pharmacokinetics of high-dose tigecycline in patients with ventilator-associated pneumonia support the use of high dose [38-40]. Thus, high-dose tigecycline may be an option for pulmonary infections as well as systemic infections as it safely increases plasma and pulmonary concentrations and was reported to have better outcomes in a retrospective study [41]. High-dose tigecycline rather than conventional dosing for CRAB is preferred.

\section{Eravacycline}

Eravacyline is a novel synthetic halogenated tetracycline class antibiotic approved for treatment of complicated intra-abdominal infections caused by susceptible microorganisms. There are reports of in vitro activity against CRAB; however, there are no CLSI breakpoints and thus no meaningful interpretation of the susceptibilities, and clinical information as a treatment option is very limited. It has not been approved for treatment of CRAB [42].

\section{Cefiderocol}

Cefiderocol is a siderophore cephalosporin developed for the treatment of MDR gramnegative infections and approved for the treatment of complicated urinary tract infections caused by susceptible organisms. Most CRAB isolates including those with OXA-type beta lactamase are reported to be susceptible to cefiderocol [43].

In the APEKS-NP trial, high-dose extended infusion of meropenem and cefiderocol therapy produced similar results [44]. However, in the CREDIBLE-CR trial, Bassetti et al. reported a higher mortality rate in patients with CRAB who were treated with cefiderocol compared to those treated with best available therapy (mostly polymyxin based regimens), $49 \%$ vs. 18\%.[45] Further research is needed to assess the clinial activity of this agent. Cefiderocol should be used with caution for CRAB infections and only as part of combination therapy.

\section{Fosfomycin}

Fosfomycin is a member of the phosphonic antibiotics that is mostly used to treat simple urinary tract infections but may have other uses as well [46]. In recent years, there has been an increased interest in combination therapies that include intravenous fosfomycin to treat CRAB. A study of 180 patients with hospital-acquired pneumonia due to CRAB demonstrated superiority of regimens that included fosfomycin [47]. However, there are not enough data to endorse using this drug for treatment of life-threating infections unless there are no other options. Furthermore, the supplies of intravenous fosfomycin are limited.

\section{Carbapenems}

Providing high-dose extended infusion carbapenems to treat serious infections caused by MDR gram-negative organisms has been suggested especially when combined with other antibiotics. Some in vitro data suggest that using extended infusion carbapenem as a third drug with ampicillin-sulbactam and either minocycline or polymyxin may lead to bacterial eradication of CRAB $[23,48]$. However, recent evidence suggests no added benefit when combining as dual therapy with colistin compared to colistin monotherapy [49]. Further research is needed to assess the combination with other antibiotics, and carbapenems may be 
considered a third drug in other combination regiments.

\section{Rifamycin}

Rifamycin antibiotics include rifampin, rifabutin and rifapentine and inhibit bacterial ribonucleic acid (RNA) polymerase. There are data suggesting synergy between rifamycin and the polymyxins that may also reduce the emergence of resistance [50]. Three randomized trials compared the clinical outcomes of patients with CRAB infections who received colistin alone versus colistin in combination with rifampin. All three trials failed to demonstrate any significant difference in outcomes for the regimens evaluated [51-53]. The limited clinical data, known toxicities and drug-drug interactions limit the use of rifamycin for CRAB infections.

\section{Inhaled Antibiotics}

The efficacy of inhaled antibiotics for the prevention and/or treatment of bacterial pneumonia has not been established. In a pharmacokinetic-pharmacodynamic modeling study, aerosolized delivery of colistin achieved high active drug levels in epithelial lining fluid of the lungs [54]. However, three clinical trials for VAP failed to demonstrate any improved outcomes or better survival with the use of nebulized antibiotics even including a subgroup analysis of drug-resistant pathogens [55-57]. This may be due to insufficient distribution throughout the lung, use of formulations not designed for inhalation or suboptimal delivery devices [58]. Currently, there is insufficient evidence to support the use of inhaled antibiotics alone for the treatment of CRAB pneumonia. Some clinicians have used aerosolized antibiotics in addition to systemic antibiotics.

\section{Future Prospects}

There are no reliable laboratory methods distinguishing colonization from true infection. The evolving technology of whole-genome phylogenetic analysis has shown that some sequential types (ST) are associated with virulent Acinetobacter strains while other are not [59]. ST10 strains have been associated with colonization and nosocomial infection in an ICU in Vietnam and an outbreak of extensively drug-resistant infection in the USA. Another STT6SS has been associated with biofilm formation and was found to have a role in host colonization and killing of competing bacteria [59]. There are few studies distinguishing infection from colonization and correlating with clinical outcomes. It is hoped that future research will demonstrate the diagnostic utility of these promising techniques.

There is ongoing research on alternative options for the treatment of CRAB including small molecules and phage therapy, all of which are still in the investigational stage. Some small studies have been conducted but the data are inconclusive as of now $[60,61]$.

\section{Combination vs. Monotherapy}

When treating an infection such as CRAB, there is no clear superior choice of antibiotic. When treating infections that are accompanied by high mortality it is very common to combine antibiotics. In infections with limited therapeutic options most clinicians prefer the use of combination therapy with agents that are individually active against a pathogen such as CRAB. The IDSA panel noted that although there have been several observational studies and seven randomized control trials, there is no clear evidence in support of a specific monotherapy or combination therapy [18]. Thus, since there is lack of robust clinical data in support of treating with any single agent, the patient population is usually very ill and suffers from severe comorbidities, and antibiotics that may initially appear active may develop resistance, they recommend the use of at least two active agents whenever possible [18].

The panel's preferred choice of therapy is ampicillin-sulbactam (high dose when the pathogen is reported as resistant) combined with either tigecycline or minocycline.

The panel is against the combination of carbapenem and polymyxin without the 
addition of a third agent ampicillin-sulbactam, which has some supportive data [23]. The panel also recommended against using fosfomycin or rifampin as part of the combinations. There is not enough evidence regarding the use of cefdericol as either a single agent or as part of a combination therapy.

\section{CONCLUSIONS}

In this narrative review, we have described the difference between colonization and infection in patients who have a positive CRAB isolate. We reviewed the current available treatments and suggest that a combination therapy is the best option based on high-dose ampicillin-sulbactam and another agent.

\section{ACKNOWLEDGEMENTS}

Funding. No funding or sponsorship was received for this study or publication of this article.

Authorship. All named authors meet the International Committee of Medical Journal Editors (ICMJE) criteria for authorship for this article, take responsibility for the integrity of the work as a whole, and have given their approval for this version to be published.

Author contributions. Carmi Bartal performed the literature search; Carmi Bartal, Kenneth Rolston and Lior Nesher created the table and figure and wrote the manuscript.

Disclosures. Carmi Bartal and Kenneth Rolston have nothing to declare; Lior Nesher has given educational lectures at conferences and participated in advisory boards paid for by Pfizer, Merck and Gilead, none of which relate to the topic of this manuscript.

Compliance with Ethics Guidelines. This article is based on previously conducted studies and does not contain any new studies with human participants or animals performed by any of the authors.

Open Access. This article is licensed under a Creative Commons Attribution-NonCommercial 4.0 International License, which permits any non-commercial use, sharing, adaptation, distribution and reproduction in any medium or format, as long as you give appropriate credit to the original author(s) and the source, provide a link to the Creative Commons licence, and indicate if changes were made. The images or other third party material in this article are included in the article's Creative Commons licence, unless indicated otherwise in a credit line to the material. If material is not included in the article's Creative Commons licence and your intended use is not permitted by statutory regulation or exceeds the permitted use, you will need to obtain permission directly from the copyright holder. To view a copy of this licence, visit http://creativecommons.org/licenses/by$\mathrm{nc} / 4.0 /$.

\section{REFERENCES}

1. Tacconelli E, Carrara E, Savoldi A, Harbarth S, Mendelson M, Monnet DL, et al. WHO pathogens priority list working group. Discovery, research, and development of new antibiotics: the WHO priority list of antibiotic-resistant bacteria and tuberculosis. Lancet Infect Dis. 2018;18(3):318-27. https://doi. org/10.1016/S1473-3099(17)30753-3.

2. Albrecht MC, Griffith ME, Murray CK, Chung KK, Horvath EE, Ward JA, et al. Impact of Acinetobacter infection on the mortality of burn patients. J Am Coll Surg. 2006;203(4):546-50. https://doi.org/10. 1016/j.jamcollsurg.2006.06.013. [Erratum in: J Am Coll Surg. 2007;204(1):191.]

3. Vincent JL, Rello J, Marshall J, Silva E, Anzueto A, Martin CD, et al. International study of the prevalence and outcomes of infection in intensive care units. JAMA J Am Med Assoc. 2009;302:2323-9.

4. Lee CR, Lee JH, Park M, Park KS, Bae IK, Kim YB, et al. Biology of Acinetobacter baumannii: pathogenesis, antibiotic resistance mechanisms, and prospective treatment options. Front Cell Infect Microbiol. 2017;7:55. 
5. Weiner LM, Webb AK, Limbago B, Dudeck MA, Patel J, Kallen AJ, et al. Antimicrobial-resistant pathogens associated with healthcare-associated infections: summary of data reported to the National Healthcare Safety Network at the Centers for Disease Control and Prevention, 2011-2014. Infect Control Hosp Epidemiol. 2016;37:1288-301.

6. Martín-Aspas A, Guerrero-Sánchez FM, García-Colchero F, Rodríguez-Roca S, Girón-González JA. Differential characteristics of Acinetobacter baumannii colonization and infection: risk factors, clinical picture, and mortality. Infect Drug Resist. 2018;11: 861-72.

7. Leung WS, Chu CM, Tsang KY, Lo FH, Lo KF, Ho PL. Fulminant community-acquired Acinetobacter baumannii pneumonia as a distinct clinical syndrome. Chest. 2006;129:102-9.

8. Garnacho-Montero J, Ortiz-Leyba C, Jiménez-Jiménez FJ, Barrero-Almodóvar AE, García-Garmendia JL, Bernabeu-Wittell M, et al. Treatment of multidrug-resistant Acinetobacter baumannii ventilatorassociated pneumonia (VAP) with intravenous colistin: a comparison with imipenem-susceptible VAP. Clin Infect Dis. 2003;36:1111-8.

9. Garnacho-Montero J, Ortiz-Leyba C, FernándezHinojosa E, Aldabó-Pallás T, Cayuela A, MarquezVácaro JA, et al. Acinetobacter baumannii ventilatorassociated pneumonia: Epidemiological and clinical findings. Intensive Care Med. 2005;31:649-55.

10. Koulenti D, Tsigou E, Rello J. Nosocomial pneumonia in 27 ICUs in Europe: perspectives from the EU-VAP/CAP study. Eur J Clin Microbiol Infect Dis. 2017;36(11):1999-2006. https://doi.org/10.1007/ s10096-016-2703-z.

11. Gaynes R, Edwards JR. Overview of nosocomial infections caused by gram-negative bacilli. Clin Infect Dis. 2005;41:848-54.

12. Sunenshine $\mathrm{RH}$, Wright MO, Maragakis LL, Harris $\mathrm{AD}$, Song X, Hebden J, et al. Multidrug-resistant Acinetobacter infection mortality rate and length of hospitalization. Emerg Infect Dis. 2007;13:97-103.

13. Adler BL, Krausz A, Friedman AJ. Acinetobacter baumannii emerging as a multidrug-resistant skin and soft-tissue pathogen: parallels to methicillin-resistant Staphylococcus aureus. JAMA Dermatol. 2014;150(8):905-6.

14. Sebeny PJ, Riddle MS, Petersen K. Acinetobacter baumannii skin and soft-tissue infection associated with war trauma. Clin Infect Dis. 2008;47(4):444-9.

15. Gradon JD, Chapnick EK, Lutwick LI. Infective endocarditis of a native valve due to acinetobacter: case report and review. Clin Infect Dis. 1992;14: 1145-8.

16. Korinek AM, Baugnon T, Golmard JL, Van Effenterre R, Coriat P, Puybasset L. Risk factors for adult nosocomial meningitis after craniotomy: role of antibiotic prophylaxis. Neurosurgery. 2006;59: 126-32.

17. Miller J. Acinetobacter as a causative agent in preseptal cellulitis. Optometry. 2005;76:176-80.

18. Tamma PD, Aitken SL, Bonomo RA, Mathers AJ, van Duin D, Clancy CJ. Infectious Diseases Society of America Guidance on the Treatment of AmpC $\beta$ lactamase-producing enterobacterales, carbapenem-resistant Acinetobacter baumannii, and Stenotrophomonas maltophilia infections. Clin Infect Dis. 2021.

19. Penwell WF, Shapiro AB, Giacobbe RA, Gu RF, Gao $\mathrm{N}$, Thresher J, et al. Molecular mechanisms of sulbactam antibacterial activity and resistance determinants in Acinetobacter baumannii. Antimicrob Agents Chemother. 2015;59:1680-9.

20. Liu J, Shu Y, Zhu F, Feng B, Zhang Z, Liu L, et al. Comparative efficacy and safety of combination therapy with high-dose sulbactam or colistin with additional antibacterial agents for multiple drugresistant and extensively drug-resistant Acinetobacter baumannii infections: a systematic review and network. J Glob Antimicrob Resist. 2021;24:136-47.

21. Jung SY, Lee SH, Lee SY, Yang S, Noh H, Chung EK, et al. Antimicrobials for the treatment of drug-resistant Acinetobacter baumannii pneumonia in critically ill patients: a systemic review and Bayesian network meta-analysis. Crit Care Crit Care. 2017;21:1-5.

22. Betrosian AP, Frantzeskaki F, Xanthaki A, Georgiadis G. High-dose ampicillin-sulbactam as an alternative treatment of late-onset VAP from multidrug-resistant Acinetobacter baumannii. Scand J Infect Dis. 2007;39:38-43.

23. Lenhard JR, Smith NM, Bulman ZP, Tao X, Thamlikitkul V, Shin BS, et al. High-dose ampicillin-sulbactam combinations combat polymyxin-resistant Acinetobacter baumannii in a hollow-fiber infection model. Antimicrob Agents Chemother. 2017;61: e01268-e1316.

24. Levin AS, Barone AA, Penço J, Santos MV, Marinho IS, Arruda EAG, et al. Intravenous colistin as therapy for nosocomial infections caused by multidrugresistant Pseudomonas aeruginosa and Acinetobacter baumannii. Clin Infect Dis. 1999;28:1008-11.

25. Florescu DF, Qiu F, McCartan MA, Mindru C, Fey PD, Kalil AC. What is the efficacy and safety of 
colistin for the treatment of ventilator-associated pneumonia? A systematic review and meta-regression. Clin Infect Dis. 2012;54:670-80.

26. Garnacho-Montero J, Timsit JF. Managing Acinetobacter baumannii infections. Curr Opin Infect Dis. 2019;32:69-76.

27. Vardakas KZ, Mavroudis AD, Georgiou M, Falagas ME. Intravenous colistin combination antimicrobial treatment vs. monotherapy: a systematic review and meta-analysis. Int J Antimicrob Agents. 2018;21:535-47.

28. Wagenlehner F, Lucenteforte E, Pea F, Soriano A, Tavoschi L, Steele VR, et al. Systematic review on estimated rates of nephrotoxicity and neurotoxicity in patients treated with polymyxins. Clin Microbiol Infect. 2021. https://doi.org/10.1016/j.cmi.2020. 12.009 .

29. Cheah SE, Wang J, Nguyen VT, Turnidge JD, Li J, Nation RL. New pharmacokinetic/pharmacodynamic studies of systemically administered colistin against Pseudomonas aeruginosa and Acinetobacter baumannii in mouse thigh and lung infection models: smaller response in lung infection. J Antimicrob Chemother. 2015;70:3291-7.

30. Qureshi ZA, Hittle LE, O'Hara JA, Rivera JI, Syed A, Shields RK, et al. Colistin-resistant Acinetobacter baumannii: beyond carbapenem resistance. Clin Infect Dis. 2015;60:1295-303.

31. López-Rojas R, McConnell MJ, Jiménez-Mejías ME, Domínguez-Herrera J, Fernández-Cuenca F, Pachón J. Colistin resistance in a clinical Acinetobacter baumannii strain appearing after colistin treatment: effect on virulence and bacterial fitness. Antimicrob Agents Chemother. 2013;57:4587-9.

32. Denys GA, Callister SM, Dowzicky MJ. Antimicrobial susceptibility among gram-negative isolates collected in the USA between 2005 and 2011 as part of the Tigecycline Evaluation and Surveillance Trial (T.E.S.T.). Ann Clin Microbiol Antimicrob. 2013;12: $1-10$.

33. Greig SL, Scott LJ. Intravenous minocycline: a review in acinetobacter infections. Drugs. 2016;76: 1467-76.

34. Ritchie DJ, Garavaglia-Wilson A. A review of intravenous minocycline for treatment of multidrug-resistant acinetobacter infections. Clin Infect Dis. 2014;59:S374-80.

35. ZhaneL GG, Karlowsky JA, Rubinstein E, Hoban D. Tigecycline: a novel glycylcycline antibiotic. Expert Rev Anti Infect Ther. 2006;4:9-25.
36. Ni W, Han Y, Zhao J, Wei C, Cui J, Wang R, et al. Tigecycline treatment experience against multidrug-resistant Acinetobacter baumannii infections: a systematic review and meta-analysis. Int $\mathrm{J}$ Antimicrob Agents. 2016;47:107-16.

37. Mei H, Yang T, Wang J, Wang R, Cai Y. Efficacy and safety of tigecycline in treatment of pneumonia caused by MDR Acinetobacter baumannii: a systematic review and meta-analysis. J Antimicrob Chemother. 2019;74:3423-31.

38. Zha L, Pan L, Guo J, French N, Villanueva EV, Tefsen $B$. Effectiveness and safety of high dose tigecycline for the treatment of severe infections: a systematic review and meta-analysis. Adv Ther. 2020;37:1049-64.

39. De Pascale G, Montini L, Pennisi MA, Bernini V, Maviglia R, Bello G, et al. High dose tigecycline in critically ill patients with severe infections due to multidrug-resistant bacteria. Crit Care. 2014;18: $1-9$.

40. Dimopoulos G, Almyroudi MP, Kapralos I, Apostolopoulou O, Flevari A, Nicolau DP, et al. Intrapulmonary pharmacokinetics of high doses of tigecycline in patients with ventilator-associated pneumonia. Int J Antimicrob Agents. 2022;59: 106487.

41. De Pascale G, Lisi L, Ciotti GMP, Vallecoccia MS, Cutuli SL, Cascarano L, et al. Pharmacokinetics of high-dose tigecycline in critically ill patients with severe infections. Ann Intensive Care. 2020;10:1-9.

42. Morrissey I, Olesky M, Hawser S, Lob SH, Karlowsky JA, Corey GR, et al. In vitro activity of eravacycline against gram-negative bacilli isolated in clinical laboratories worldwide from 2013 to 2017. Antimicrob Agents Chemother. 2020;64(3): e01699-19. https://doi.org/10.1128/AAC.01699-19.

43. Kazmierczak KM, Tsuji M, Wise MG, Hackel M, Yamano Y, Echols R, et al. In vitro activity of cefiderocol, a siderophore cephalosporin, against a recent collection of clinically relevant carbapenemnon-susceptible Gram-negative bacilli, including serine carbapenemase- and metallo- $\beta$-lactamaseproducing isolates (SIDERO-WT-2014). Int J Antimicrob Agents. 2019;53:177-84.

44. Wunderink RG, Matsunaga Y, Ariyasu M, Clevenbergh P, Echols R, Kaye KS, et al. Cefiderocol versus high-dose, extended-infusion meropenem for the treatment of Gram-negative nosocomial pneumonia (APEKS-NP): a randomised, double-blind, phase 3, non-inferiority trial. Lancet Infect Dis. 2021;21: 213-25.

45. Bassetti M, Echols R, Matsunaga Y, Ariyasu M, Doi $\mathrm{Y}$, Ferrer R, et al. Efficacy and safety of cefiderocol or 
best available therapy for the treatment of serious infections caused by carbapenem-resistant Gramnegative bacteria (CREDIBLE-CR): a randomised, open-label, multicentre, pathogen-focused, descriptive, phase 3 trial. Lancet Infect Dis. 2021;21:226-40.

46. Falagas ME, Giannopoulou KP, Kokolakis GN, Rafailidis PI. Fosfomycin: use beyond urinary tract and gastrointestinal infections. Clin Infect Dis. 2008;46:1069-77.

47. Russo A, Bassetti M, Bellelli V, Bianchi L, Marincola Cattaneo F, Mazzocchetti S, et al. Efficacy of a fosfomycin-containing regimen for treatment of severe pneumonia caused by multidrug-resistant Acinetobacter baumannii: a prospective, observational study. Infect Dis Ther Infect Dis Ther. 2021;10:187-200.

48. Abdul-Mutakabbir JC, Yim J, Nguyen L, Maassen PT, Stamper K, Shiekh Z, et al. In vitro synergy of colistin in combination with meropenem or tigecycline against carbapenem-resistant Acinetobacter baumannii. Antibiotics (Basel). 2021;10(7):880. https://doi.org/10.3390/antibiotics10070880.

49. Paul M, Daikos GL, Durante-Mangoni E, Yahav D, Carmeli Y, Benattar YD, et al. Colistin alone versus colistin plus meropenem for treatment of severe infections caused by carbapenem-resistant Gramnegative bacteria: an open-label, randomised controlled trial. Lancet Infect Dis. 2018;18:391-400.

50. Cheng J, Yan J, Reyna Z, Slarve M, Lu P, Spellberg B, et al. Synergistic rifabutin and colistin reduce emergence of resistance when treating Acinetobacter baumannii. Antimicrob Agents Chemother. 2021;65(4):e0220420. https://doi.org/10.1128/AAC .02204-20.

51. Durante-Mangoni E, Signoriello G, Andini R, Mattei A, De Cristoforo M, Murino P, et al. Colistin and rifampicin compared with colistin alone for the treatment of serious infections due to extensively drug-resistant Acinetobacter baumannii: a multicenter, randomized clinical trial. Clin Infect Dis. 2013;57:349-58.

52. Aydemir H, Akduman D, Piskin N, Comert F, Horuz $\mathrm{E}$, Terzi A, et al. Colistin vs. the combination of colistin and rifampicin for the treatment of carbapenem-resistant Acinetobacter baumannii ventilator-associated pneumonia. Epidemiol Infect. 2013;141:1214-22.

53. Park HJ, Cho JH, Kim HJ, Han SH, Jeong SH, Byun MK. Colistin monotherapy versus colistin/rifampicin combination therapy in pneumonia caused by colistin-resistant Acinetobacter baumannii: a randomised controlled trial. J Glob Antimicrob Resist. 2019;17:66-71.
54. Boisson $\mathrm{M}$, Jacobs $\mathrm{M}$, Grégoire $\mathrm{N}$, Gobin $\mathrm{P}$, Marchand S, Couet W, et al. Comparison of intrapulmonary and systemic pharmacokinetics of colistin methanesulfonate (CMS) and colistin after aerosol delivery and intravenous administration of CMS in critically ill patients. Antimicrob Agents Chemother. 2014;58(12):7331-9. https://doi.org/ 10.1128/AAC.03510-14.

55. Rattanaumpawan $P$, Lorsutthitham J, Ungprasert $P$, Angkasekwinai N, Thamlikitkul V. Randomized controlled trial of nebulized colistimethate sodium as adjunctive therapy of ventilator-associated pneumonia caused by Gram-negative bacteria. J Antimicrob Chemother. 2010;65(12):2645-9. https://doi.org/10.1093/jac/dkq360.

56. Kollef MH, Ricard JD, Roux D, Francois B, Ischaki E, Rozgonyi $\mathrm{Z}$, et al. A randomized trial of the amikacin fosfomycin inhalation system for the adjunctive therapy of gram-negative ventilator-associated pneumonia: IASIS trial. Chest. 2017;151(6):1239-46. https://doi.org/10.1016/j. chest.2016.11.026.

57. Niederman MS, Alder J, Bassetti M, Boateng F, Cao $\mathrm{B}$, Corkery K, et al. Inhaled amikacin adjunctive to intravenous standard-of-care antibiotics in mechanically ventilated patients with Gram-negative pneumonia (INHALE): a double-blind, randomised, placebo-controlled, phase 3 , superiority trial. Lancet Infect Dis. 2020;20(3):330-40. https:// doi.org/10.1016/S1473-3099(19)30574-2.

58. Rouby JJ, Bouhemad B, Monsel A, Brisson H, Arbelot C, Lu Q. Nebulized Antibiotics Study Group. Aerosolized antibiotics for ventilator-associated pneumonia: lessons from experimental studies. Anesthesiology. 2012;117(6):1364-80. https://doi. org/10.1097/ALN.0b013e3182755d7a.

59. Meumann EM, Anstey NM, Currie BJ, Piera KA, Kenyon JJ, Hall RM, et al. Genomic epidemiology of severe community-onset Acinetobacter baumannii infection. Microb Genom. 2019;5(3):e000258. https://doi.org/10.1099/mgen.0.000258.

60. Broncano-Lavado A, Santamaría-Corral G, Esteban J, García-Quintanilla M. Advances in bacteriophage therapy against relevant multidrug-resistant pathogens. Antibiotics (Basel). 2021;10(6):672. https://doi.org/10.3390/antibiotics10060672.

61. Choi J, Jang A, Yoon YK, Kim Y. Development of novel peptides for the antimicrobial combination therapy against carbapenem-resistant Acinetobacter baumannii infection. Pharmaceutics. 2021;13(11): 1800. https://doi.org/10.3390/pharmaceutics13111 800. 